\title{
Interplay between geometry and flow distribution in an airway tree
}

\author{
B. Mauroy ${ }^{1}$, M. Filoche ${ }^{2}$, J. S. Andrade Jr. ${ }^{1,3}$, and B. Sapoval ${ }^{1,2}$ \\ ${ }^{1}$ Centre de Mathématiques et de leurs Applications, CNRS, Ecole Normale Supérieure de Cachan, 94235 Cachan, France \\ ${ }^{2}$ Laboratoire de Physique de la Matière Condensée, CNRS, Ecole Polytechnique, 91128 Palaiseau, France \\ ${ }^{3}$ Departamento de Física, Universidade Federal do Ceará, 60451-970 Fortaleza, Ceará, Brazil
}

(November 17, 2018)

\begin{abstract}
Uniform flow distribution in a symmetric volume can be realized through a symmetric branched tree. It is shown here however, by $3 \mathrm{D}$ numerical simulation of the Navier-Stokes equations, that the flow partitioning can be highly sensitive to deviations from exact symmetry if inertial effects are present. The flow asymmetry is quantified and found to depend on the Reynolds number. Moreover, for a given Reynolds number, we show that the flow distribution depends on the aspect ratio of the branching elements as well as their angular arrangement. Our results indicate that physiological variability should be severely restricted in order to ensure adequate fluid distribution through a tree.
\end{abstract}

PACS number : 47.60.+i, 87.19.Uv

The problem of fluid flow in a branching geometry appears in many physical, geological, chemical and biological systems. Examples include catalysis, flow through porous media, blood circulation and respiration. When studying transport in any of these systems, a common objective is to understand the mechanisms that govern the flow partitioning at the interconnections level. Until recently, it has been generally assumed that the use of Darcy's law should be sufficient to describe the propagation of flow through branched structures. Such a relation corresponds to the linear dependence between flow and pressure drop, $Q \propto \Delta P$, which is strictly valid at small Reynolds number. Regardless of this limitation, a large number of studies have been based on this approximation. In the context of porous media, for instance, a simple paradigm to represent flow through the pore space is a network of bifurcating and merging channels where the transport of fluid is analogous to the distribution of electrical currents in a resistor network. However these models can only predict a perfectly uniform and synchronous flow distribution through airways bifurcations [1]. A major problem in modeling of flow through trees arises from the fact that, due to inertial effects, Darcy's law breaks down as a phenomenological description for large Reynolds numbers. Even at moderate Reynolds, the inertial non-linearities become relevant as compared to viscous effects.

Unambiguous experimental and numerical evidences of inertial effects have been observed in several studies on flow though branched structures, with special emphasis on the bronchial tree [2-14]. Such phenomena exists in real lungs but they are more simple to study in a symmetric geometry $[15,16]$. In particular, in order to irrigate uniformly a symmetric volume it is easy to show, through the following collage argument, that this is ensured by a symmetric tree. Suppose that an asymmetric tree feeds a volume which has a plane of symmetry. If the tree is asymmetric the flow will be different in the two parts of the volume which are symmetrical. Then one can replace the tree with a non-uniform flow by the symmetry image of the more efficient region. The new tree, which is now symmetric, is more efficient for flow distribution.

In the Poiseuille approximation, the only way to have perfect symmetry is to work with an equivalent resistor network that is symmetric. In other words, at each bifurcation the daughter branches should be exactly identical irrespectively of their real geometrical arrangement. This might not be true if inertial effects are present. It should be recalled that, as the lung is a succession of branch bifurcations, the final flow distribution can be represented by a multiplicative process. In consequence, even a rather small asymmetry could lead to a strong inhomogeneity of the flow distribution [17]. Because the geometrical arrangement of the bronchial tree of mammals is always subjected to some physiological variability [18], it appears natural to question whether a small modification of the structure disturbs the distribution of fluid flow.

The purpose of this work is to investigate how the tree geometry influences the flow in order to shed some light on the optimal aspect of the bronchial tree for distributing air uniformly in the lung volume. The direct 3D numerical solution of the Navier-Stokes equations is by far the most practical way to elucidate this problem. The simplified tree model used here is shown in Fig. 1. It consists in a 3-dimensional cascade of cylinders branching through two bifurcations. Each bifurcation $\mathrm{ABC}$ or $\mathrm{BDE}$ or CFG, is coplanar as found approximately in real lungs. The bifurcation geometries are modeled in such a way to minimize geometrical singularities as shown in Fig. 2. For simplicity, we assume that the radii of the tubes decrease with a factor $2^{-1 / 3}$ at each bifurcation [19] and choose the branching angle to be $45^{\circ}$.

The mathematical description for the detailed fluid mechanics in the branched structure is based on the steadystate form of the continuity and Navier-Stokes equations 
for mass and momentum conservation [20]

$$
\begin{gathered}
\nabla \cdot \mathbf{u}=0 \\
\rho \mathbf{u} \cdot \nabla \mathbf{u}=-\nabla p+\mu \nabla^{2} \mathbf{u},
\end{gathered}
$$

where $\mathbf{u}$ and $p$ are the local velocity and pressure fields, respectively. The diameter of the first tube is equal to $2 \mathrm{~cm}$, corresponding approximately to the diameter of the human trachea. The fluid is air with viscosity $\mu=$ $1.785 \times 10^{-5} \mathrm{~kg} \mathrm{~m}^{-1} \mathrm{~s}^{-1}$ and density $\rho=1.18 \mathrm{~kg} \mathrm{~m}^{-3}$, and the flow is considered to be incompressible. Nonslip boundary conditions are imposed at the tube walls (Dirichlet condition $\mathbf{u}=0$ ) and the velocity at the entrance A is parabolic. The outlets are free with the same reference pressure and $\partial \mathbf{u} / \partial n=0$. Equations (1) and (2) are solved using finite elements [21]. For all simulations, the relative conservation error is smaller than $3 \%$.

The parameters governing the flow are the bronchi aspect ratio (length to diameter ratio of the tubes) $L / D$, the rotation angle $\alpha$ between successive bifurcations, and the Reynolds number, $R e \equiv \rho D V / \mu$, where $V$ is the mean velocity at the entrance. The reference angle $\alpha=0^{\circ}$ corresponds to a coplanar tree. The flow asymmetry is defined as

$$
\Sigma(\alpha, L / D) \equiv\left|\frac{q_{1}-q_{2}}{q_{1}+q_{2}}\right|,
$$

where $q_{1}$ and $q_{2}$ are the outflows at $(\mathrm{D}, \mathrm{G})$ and $(\mathrm{E}, \mathrm{F})$ branches, respectively. We perform simulations for several values of $\alpha, L / D$ and $R e$ to find their influence on the flow partitioning $\Sigma$. Note that the air velocity at the entrance of human lungs range from $1 \mathrm{~m} / \mathrm{s}$ at rest $(R e \approx 1200)$ to $10 \mathrm{~m} / \mathrm{s}$ for the condition of very hard exercise $(R e \approx 12000)$ [19]. Due to the number of parameters governing the flow and the computation time for each set of parameters, we first discuss the dependence of the flow asymmetry on the geometry for a fixed Reynolds value, namely, $R e=1200$. This corresponds to the human inspiration state at rest.

The results are shown in Fig. 3. The main result is that, whatever the conditions, the behavior of $\Sigma$ around the minimum is not parabolic. Even a small departure from geometrical symmetry can cause a non negligible flow disturbance. For a given value of $L / D$, the disturbance increases with the deviation of the rotation angle from $90^{\circ} . \Sigma$ is therefore maximum for a planar tree and, for a fixed $\alpha$ value, it decreases with increasing aspect ratio.

There are then two facts to interpret. First, why the flow is influenced by breaking the symmetry only. Seconds why this effect is attenuated for long branches or large aspect ratios. The first fact can be understood by considering the velocity distribution in a cut of the secondary branch B shown in Fig. 4. The flow keeps the symmetry of the ABC bifurcation plane but, due to inertia, the high velocity regions are drifted vertically and an M-shape type of distribution is observed [9]. This shape governs the flow partitioning at the second bifurcation. Note that if the branches B and C are long enough and for small $R e$, the profile should tend to a parabolic type. As a consequence, the distribution shown in Fig. 3 will progressively change along the second generation branch. It is because the branch length is too short that the granddaughter branches can capture the asymmetry seen in Fig. 4. This provides a qualitative answer for the second question. The position of the intersection relatively to the M-shape is then the key for asymmetry. For example in Fig. 4, the branch $\mathrm{E}$ obviously receives more flow than branch $\mathrm{D}$. It is also clear that, if $\alpha=90^{\circ}$, the flow symmetry is restored for any value of $L / D$.

The dependence of the flow asymmetry as a function of the Reynolds number is shown in Fig. 5. A strong increase of $\Sigma$ is observed up to $R e \approx 250$ followed by a region of weaker dependence. This type of behavior has been previously reported for $2 \mathrm{D}$ flow in trees comprising more than two generations of branches $[9,10]$. It is remarkable that the onset at $R e \approx 250$ is approximately the same whatever the angle $\alpha$. This is a clear indication that, at the entrance of the second bifurcation, the velocity profile reaches the same pattern for a given $R e$ value. Again, the final asymmetry of the distribution of flow is a result of the inertial effects originated from the first bifurcation. All these arguments are illustrated in Fig. 6, where the contour plots of the velocity fields are shown at the entrance of the second bifurcation. At large $R e$, the M-shape is revealed and, as expected, the lower the $R e$, the closer the profile is to parabolic flow. The smaller variation of $\Sigma$ for $R e>250$ can be explained by the presence of a secondary flow [13].

Some implications of our results are noteworthy. If the inertial effects observed here are present in a larger tree, the relative flows delivered to the outlets of this structure may become strongly non-uniform. This broadness in the flow distribution is a typical signature of a multiplicative process [22], where an observable can be viewed as a "grand process" depending on the successful completion of a number $n$ of independent "subprocesses". It is then possible to associate the flow at each branch with a probability $p_{i}$, so that the flow at a given outlet $k$ be $q_{k} \propto p_{1} p_{2} \cdots p_{n}$, where $i=1,2,3, \cdots, n$ corresponds to the set of branches constituting the pathway going from the entrance to the exit $k$. It can be easily demonstrated that, if the $p_{i}$ 's are independent variables and $n$ large, the distribution of $q_{k}$ should be approximately log-normal. Furthermore, this distribution might mimic a power-law if its dispersion is sufficiently large [22]. Note that this situation is that of the human bronchial tree (where $L / D$ is close to 3 ) even at rest. In this case $R e \approx 1200$ and the multiplicative process due to inertia can propagate further down in the tree. If we consider that these effects 
only disappear for $R e$ less than 100 and that the local Reynolds decreases by a factor of $2^{2 / 3}$ at each generation, we obtain that the flow asymmetry can be significant up to the 6 th generation of the bronchial tree under rest conditions [23].

In conclusion, we have investigated the effect of inertia on fluid flow through three-dimensional rigid branched structures by direct numerical simulation of the NavierStokes equations. It has been found that for trees with 3 generations of cylindrical conduits, the flow distribution at the outlets strongly depends on the Reynolds number and on the geometry of the ramified structure. Moreover, our simulations indicate that the flow imbalance throughout the tree is highly sensitive to the aspect ratio $L / D$ of its cylindrical units and to the variation of the rotation angle $\alpha$ between successive bifurcations. While a uniform distribution of flows at the outlets of the third generation branches is always obtained for $\alpha=90^{\circ}$, our calculations show that a small deviation from this geometrical configuration is capable to induce a large asymmetry on the flow. Note that the presence of long branches would lead to purely axi-symmetric parabolic profiles and flow symmetry. However, long tubes exhibit large hydrodynamic resistance (proportional to $L / D^{4}$ ). It is therefore not surprising that in real lungs $L / D \approx 3$ and $\alpha \approx 90^{\circ}$.

Finally, our results suggest that small deviations from the "best" structure should have the same type of consequences in the real (asymmetric) lung, namely, strong dependence on geometry and Reynolds number. In particular, the flow distribution at rest and exercise might be significantly different. These results could also help to better understand lung morphology. It has been argued [24] that the asymmetric structure of the lung is solely due to geometrical constraints. Our study indicates that the inertial effects plays also an important role in air distribution. In other words, the asymmetry of the bronchial tree is determined not only by geometrical constraints but also by the existence of inertial effects. Of course, if the flow distribution is found uniform although the geometry is "imperfect", the following question would naturally arise [25]: what are the physiological regulation mechanisms that can compensate the flow non-uniformity due to inertial effects? In addition, the fluid dynamics studied here is certainly relevant to understand particle deposition in the airway tree [13], a problem of crucial importance both from the physiologic and the therapeutic points of view.

We thank CNPq, CAPES, COFECUB and FUNCAP for support. The Centre de Mathématiques et de leurs Applications and the Laboratoire de Physique de la Matière Condensée are "Unité Mixte de Recherches du Centre National de la Recherche Scientifique" no. 8536 and 7643.
[1] T.J. Pedley, R.C. Shroter and M.F. Sudlow, Respir. Physiol. 9, 387 (1970).

[2] J. B. Grotberg, Annu. Rev. Biomed. Eng. 3, 421 (2001).

[3] B. Snyder, D. R. Dantzker, and M. J. Jaeger, J. Appl. Physiol. 51, 598 (1981).

[4] B. Snyder and M. J. Jaeger, J. Appl. Physiol. 54, 749 (1983).

[5] A. S. Slutsky, G. G. Berdine, and J. M. Drazen, J. Appl. Physiol. 49, 417 (1980).

[6] D. J. Isabey, J. Biomech. 15, 395 (1982).

[7] J. L. Allen, I. D. Frantz, and J. J. Fredberg, J. Clin. Invest. 76, 620 (1985).

[8] A. Tsuda, and J. J. Fredberg, J. Appl. Physiol. 69, 546 (1990); ibid., 69, 553 (1990).

[9] F. Wilquem and G. Degrez, J. Biomech. Eng. 119, 59 (1997).

[10] J. S. Andrade, Jr. et al., Phys. Rev. Let. 81, 926 (1998); M. P. Almeida et al., Phys. Rev. E 60, 5486 (1999).

[11] Y. Zhao, C. T. Brunskill, and B. B. Lieber, J. Biomech. Eng. 119, 52 (1997).

[12] T. B. Martonen, X. Guan, and R. M. Schreck, Inhal. Toxicol. 13, 261 (2001).

[13] J. K. Comer, C. Kleinstreuer, and Z. Zhang, J. Fluid Mech. 435, 25 (2001).

[14] H. K. Chang, and M. Paiva, Respiratory Physiology (Dekker, New York, 1989), p. 63.

[15] B. B. Mandelbrot, The Fractal Geometry of Nature (W. H. Freemann, San Francisco, 1982).

[16] W. I. Newman, D. L. Turcotte, and A. M. Gabrielov, Fractals 5, 603 (1997).

[17] B. Sapoval, Universalités et fractales (Flammarion, Paris, 1997).

[18] M. F. Shlesinger and B. J. West, Phys. Rev. Lett. 67, 2106 (1991).

[19] E. R. Weibel, The Pathway for Oxygen (Harvard Univ. Press, 1984).

[20] The steady state assumption for inspiration flow has been justified in several papers; T. J. Pedley, R. C. Schroter, and M. F. Sudlow in Bioengineering Aspects of the Lung, edited by J. B. West (Dekker, New York, 1977).

[21] We apply the solver N3S4.0 in a discretized geometry build through the code Simail, both from Simulog. Between 200000 and 350000 tetrahedral elements are used with $P^{2}$ interpolation for the velocity field and $P^{1}$ interpolation for pressure. The convergence is monitored for flow asymmetry and flow conservation.

[22] B. J. West, and M. F. Shlesinger, Int. J. Mod. Phys. B 3, 795 (1989).

[23] The situation addressed here corresponds to the functioning of the normal lung of adults. Peripheral airway closure might occur in pathological situations; B. Suki et al., Nature 368, 615 (1994) and A.-L. Barabasi et al., Phys. Rev. Lett 76, 2192 (1996).

[24] T. R. Nelson and D. K. Manchester, IEEE Trans. on Med. Imag. 7, 321 (1988).

[25] K. C. Beck and T. A. Wilson, J. Appl. Physiol. 90, 2151 (2001). 


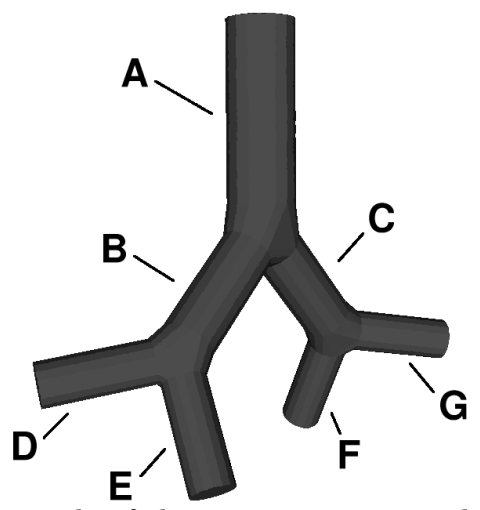

FIG. 1. Example of the tree geometry used in the simulations. The aspect ratio is $L / D=3$ and the rotation angle is $\alpha=45^{\circ}$.

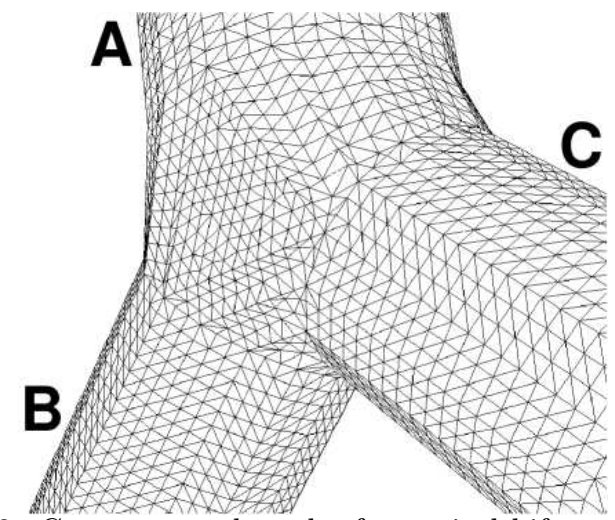

FIG. 2. Geometry and mesh of a typical bifurcation used in the simulations.

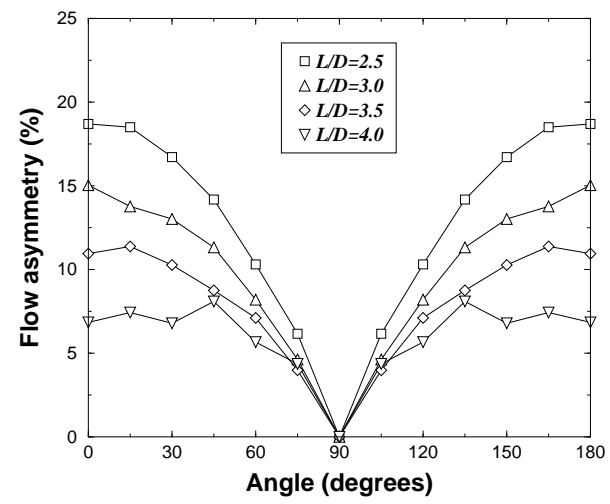

FIG. 3. Dependence of the flow asymmetry $\Sigma$ on the branching angle $\alpha$ for a fixed Reynolds value, $R e=1200$. The observed non-monotonous dependences are due to numerical uncertainties. The values of $\alpha=0^{\circ}$ and $180^{\circ}$ correspond to a planar tree. $\alpha=90^{\circ}$ represents the average value for mammalian lungs.

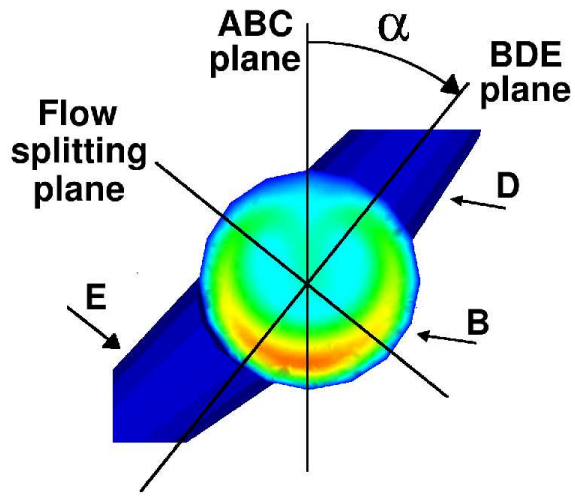

FIG. 4. The M-shape contour for $L / D=3$ and $\alpha=45^{\circ}$. The colours indicate the magnitude of the fluid velocity at the mid-length cross-section of branch $\mathrm{B}$. The velocity magnitude increases in the colour order of blue, green, yellow and red. The ternary branches D and E are shown in blue. Note the presence of a low velocity region at the center. At the plane of the second bifurcation, the entering flow is larger at the bottom. The branch $\mathrm{E}$ therefore captures a larger flow than D.

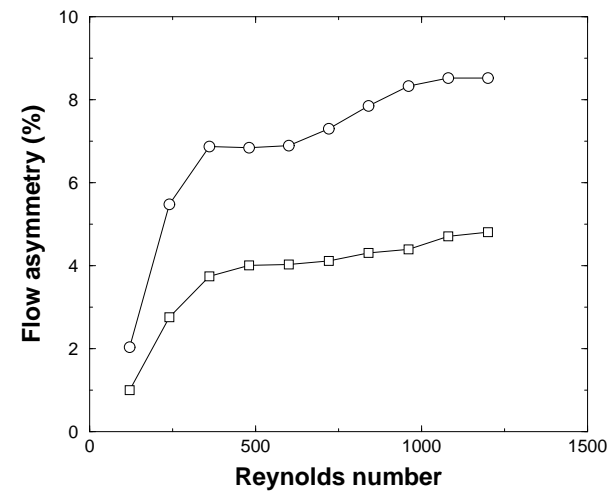

FIG. 5. Dependence of the flow asymmetry $\Sigma$ on the Reynolds number $R e$ for $L / D=3$. The circles correspond to $\alpha=60^{\circ}$ and the squares to $\alpha=75^{\circ}$.

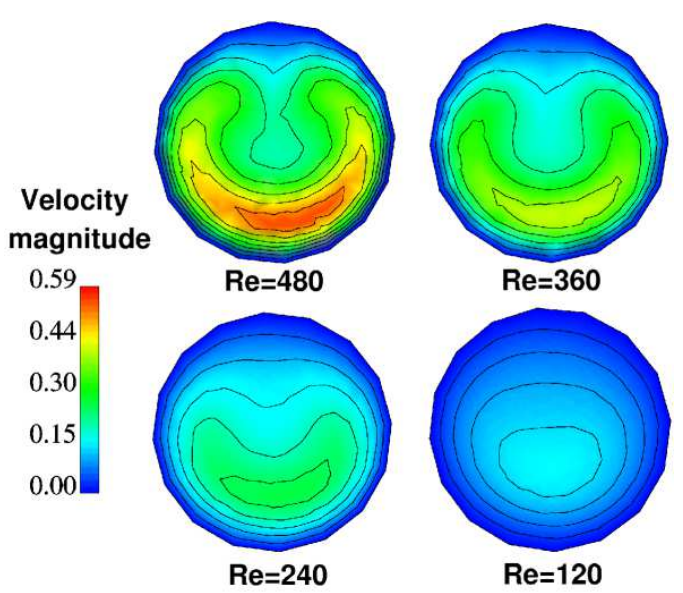

FIG. 6. Contour plot of the velocity magnitude at the cross-section of the second bifurcation for different values of $\operatorname{Re}\left(L / D=3\right.$ and $\left.\alpha=60^{\circ}\right)$. As Re increases, the profiles 4 gradually change from parabolic to M-shape. 